\title{
Physical Parameters Related to Quantify Quality of Effectiveness of Charged Particles at Lower Doses
}

\author{
Abubaker Ali Yousif, Ismail Bin Bahari, Muhamad Samudi Yasir \\ Nuclear Science Programme, School of Applied Physics, Faculty of Science and Technology, \\ University Kebangsaan Malaysia, Selagor, Malaysia \\ E-mail:alghoul75@yahoo.com \\ Received January 18, 2011; revised March 10, 2011; accepted March 15, 2011
}

\begin{abstract}
Quality factors for protons and helium particles has determined for cell inactivation using linear energy transfer. The quality has also been investigated as a function for other physical parameters, such as mean free path and effective charge for protons and helium particles, for a better interpretation to the effectiveness of these charged particles in V79 cells. Explanation of quality is clearly illustrated in terms of the average distance of energy deposition events in biological systems.
\end{abstract}

Keywords: Quality Factor, Inactivation Effect, Physical Parameters

\section{Introduction}

Investigations of the biological effects of low doses ionizing radiation is important in the application of radiological protection.

The different types of radiation has different qualities in terms of equal doses, whereby it is necessary to introduce quality factors, $\mathrm{Q}$, which are used as a multiplier yields a dose-equivalent, quality factors are allocated values which are exceed the measured RBE's relevant to end-point [1].

The quality factor from the absorbed dose is to be multiplied in order to obtain a quantity that expresses a common scale for all ionizing radiation qualities. Up to now, $\mathrm{Q}$ is related to linear energy transfer, LET, of the radiation. LET is not a good physical quality parameter to quantify the radiation effects, as such representing $Q$ in terms of LET is not an accurate method to quantify the effectiveness of ionizing radiations [2].

The relative biological effectiveness, RBE, will be unlimited quantity if the absorbed dose in of survival curves for mammalian cells approaches the zero point. This problem is normal in radiation protection that involves the low doses. The quality factors are introduced in radiation protection because of the fundamental difficulties that relates to RBE [3] and it is also because of the large variations found in many biological systems, quality factors are usually based on the RBE measurements [4].

As the same absorbed dose delivered by radiations th- rough different quality, i.e., linear energy transfer LET that has different effectiveness in causing damages, as such absorbed energy is not appropriate physical quantity for radiation protection at lower doses. On the other hand, RBEs are too complex and it's also too dependent on absorbed dose, dose rate as well as the biological end-point, and it is to be used in radiation protection [5]. The quality factor $\mathrm{Q}$ replaces $\mathrm{RBE}$ in radiation protection. While RBEs are measured values, the quality factors are established on the basis of measured RBEs. The quality factor can be viewed as an average RBE in different doses, dose rates, as well as the endpoints.

The probability of a stochastic effect does not only depend on the absorbed dose but also on the type and energy of the radiation. Some radiation doses are more effective biologically, compared to other doses. All these are taken into account by weighting the absorbed dose through a factor which is related to the quality of the radiation. A radiation weighting factor is a multiplier quantity whereby it is used to place biological effects (risks) from exposure of the different types of general radiation measure [6].

The specification of radiation quality is based on the energy deposition which has a limited value in defining the quality of ionizing radiations in creating damage [7]. The quality of ionizing radiation produces inactivation effect which is more obvious if it interpreted based on the mean free path between the primary ionizations, i.e. by determining the mean free path between the primary io- 
nizations along with the particle tracks corresponding to $2 \mathrm{~nm}$ spacing between the strands in double-stranded DNA. Anyway, the radiation quality can also determine by using microdosimetric quantities.

One contention states that the energy imparted to the biological target is not the primary factor in determining the radiation quality but it is meant as a mean free path for the charged particles that travels through the targets. The optimum mean free path was found to be about $2 \mathrm{~nm}$ related to the DNA double strand break [8].

Watt [9] has reported that if a holistic system of radiation protection and dosimetry does not require foreknowledge for the radiation quality has established, then the quality factors will become old-fashioned.

Whatever advances made in knowledge and understanding of the biological effects of ionizing radiation, the shortages continuous in limiting the ability to provide a fixed scientific basis for the radiation protection criteria [10], i.e., the ability to evaluate realistic values of the quality factor for different qualities of radiation which depends mainly in knowing the exact interaction mechanism of the ionizing radiation inside the biological targets.

In this paper, the quality factors of two types of ionizing radiations $\left({ }^{1} \mathrm{H}\right.$ and ${ }^{4} \mathrm{He}$ ) are determined as function of linear energy transfer, quality factors of these particles, in certain range of energy spectrum, are represented graphically in terms of other physical quality parameters, such as particles energy $E$, free path $\lambda$, and effective charge $Z_{\text {eff }}$ to identify the dependence of quality of effectiveness for inactivation effect on theses parameters at lower doses, and what the physical features of these representations in radiation protection.

\section{Materials and Method}

Quality factor which represents the biological effectiveness of radiation quality is determined by using the following expression [11].

$$
\begin{array}{ll}
Q(L)=1 & \text { for } L<10 \\
Q(L)=0.32 \times L-2.2 & \text { for } L: 10-100 \\
Q(L)=300 \times L^{-1 / 2} & \text { for } L>100
\end{array}
$$

Where $Q$ represents quality factor of charged particle and $\mathrm{L}$ is unrestricted linear energy transfer. In this expression of quality factor, linear energy transfer has divided for three regions.

Effective charge $\left(Z_{\text {eff }}\right)$ of proton and helium particles is calculated utilizing Equations (4) and (5) respectively.

$$
\begin{aligned}
& Z_{\text {eff }}=\left(\frac{E}{84 \times A}\right)^{1.2} \\
& Z_{\text {eff }}=\left(\frac{E}{84 \times A}\right)^{0.9}
\end{aligned}
$$

Linear primary ionization (I) represents the number of ionization events per unit track length of charged particle traversal in the irradiated entity.

Mean free path for primary ionization, $\lambda$, expresses the average distance traversed by before a specified type quality of radiation between subsequent interactions (ionizations) in the medium. Average distance $\lambda$ can get directly from simple reciprocal of I. The most effective value of $\lambda$ would represent the spacing of double strands of DNA segment in cell nucleus. This parameter has used by Cannell and Watt [12] to investigate the biophysical mechanism of fast particles effects in mammalian cells.

Physical quality parameters for every particle which are studied in this research are taken from the same experiments. Unrestricted linear energy transfer, linear primary ionization and mean free paths are interpolated using the standard values of physical parameters [13]. The physical relationships between $\mathrm{Q}$ and the different physical parameters are appraised on the type of correlations evolved between the chosen parameters.

\section{Results and Discusion}

Figure 1 shows relationship between quality factor and energy for protons. As energy of proton increases the quailty decreases dramatically. At $1700 \mathrm{keV}$, quality of protons reaches the minimum value. After that quality rises up again. The good correlation of data is fitted by the following model:

$$
Q(E)=C_{1} E+C_{2} E^{2}+C_{3}
$$

where $C_{1}=-7.59, C_{2}=2.22$,

$$
C_{3}=3.82 \text { with } R^{2}=0.90 \text {. }
$$

The same relationship between quality factor and energy for helium particles has obtained as represented in Figure 2. Quality factor inversely decreases as a function

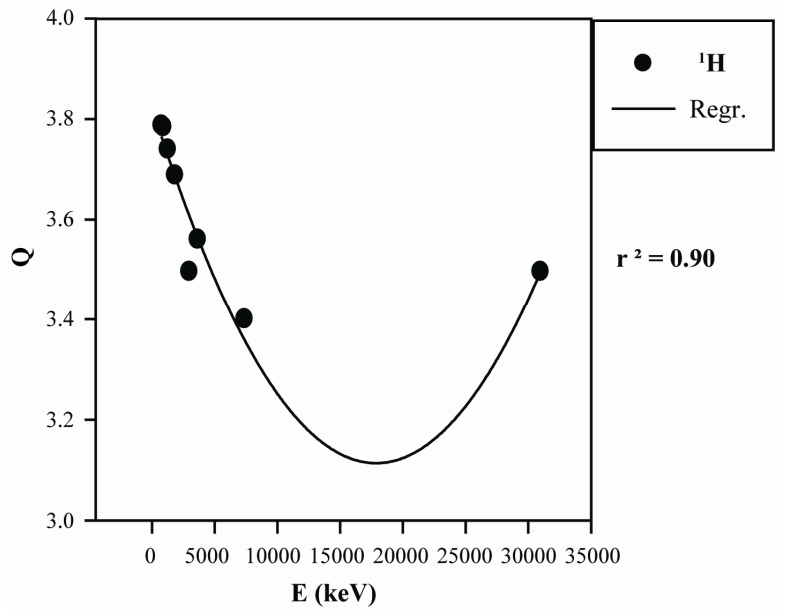

Figure 1. Relationship between quality factor and energy for protons used to irradiate V79 cells invitro. 


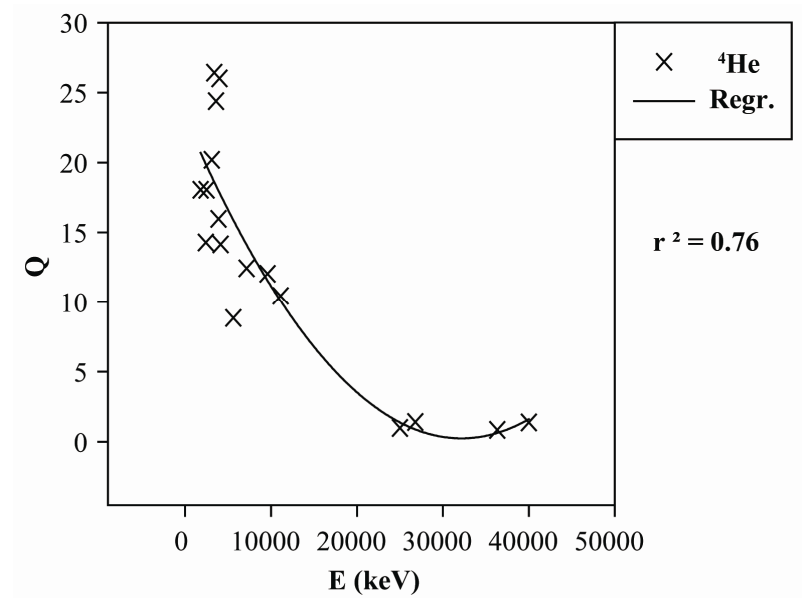

Figure 2. Relationship between quality factor and energy for helium particles used to irradiate V79 cells invitro.

of helium particles energy. That data is fitted with linear quadratic expression which given as:

$$
Q(E)=C_{4} E+C_{5} E^{2}+C_{6} E^{3}+C_{7}
$$

where $C_{4}=-7.14 \times 10^{-3}, C_{5}=8.28 \times 10^{-7}$,

$$
C_{6}=-3.23 \times 10^{-11}, C_{7}=28.32 \text {, with } R^{2}=0.76 \text {. }
$$

The relationship between quality factors and linear energy transfer for protons is presented in Figure 3. There is half bell-shape response, at the beginning, quality factor is steadily increased with increasing in LET, after certain value the quality factor went up to maximum value at $19 \mathrm{keV} / \mu \mathrm{m}$. The data has fitted by the following mathematical expression:

$$
Q(L)=C_{8} L+C_{9} L^{2}+C_{10} L^{3}+1.22
$$

where $C_{8}=-0.11, C_{9}=7.82$,

$$
C_{10}=3.08 \text {, with } R^{2}=0.99 \text {. }
$$

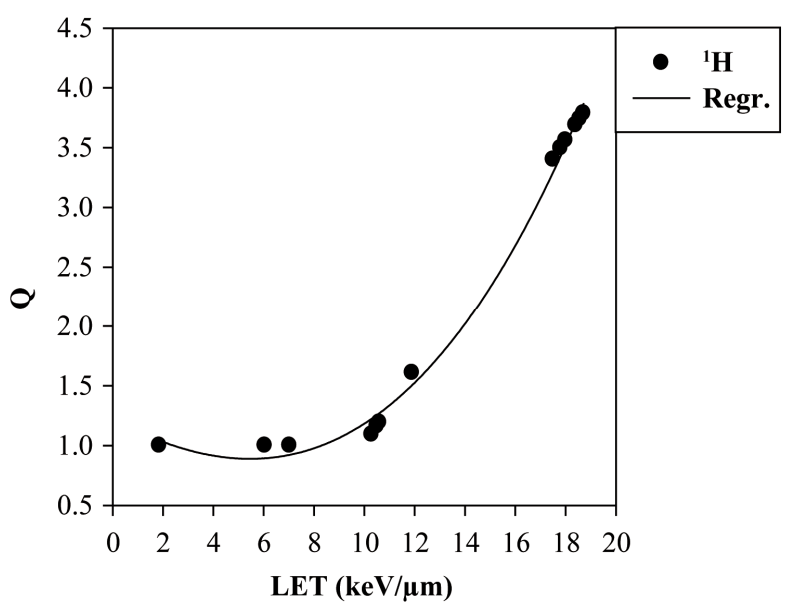

Figure 3. Relationship between quality factor and linear energy transfer for protons used to irradiate V79 cells invitro.
Figure 4 indicates the relation between quality factor and linear energy transfer for helium particles, in this relation quality factor is proportional linearly with LET. This response can be modeled as:

$$
Q(L)=C_{11} L-2.2
$$

where $C_{11}=0.32$, with $R^{2}=1$.

In Figure 5 the relation between quality factor and mean free path has been represented to better quantification of quality factor on the basis of average distances of ionization events rather than energy description parameters. As is observed, quality factor for protons decreases dramatically as mean free path increases, in this spectrum of proton energies, inactivation of V79 cells does not occur at low doses. The good representative model of the represented data is:

$$
Q(\lambda)=C_{12} \lambda+C_{13} \lambda^{2}+1.59
$$

where $C_{12}=-11 \times 10^{-2}, C_{13}=5.07 \times 10^{-3}$, with $R^{2}=$ 0.99

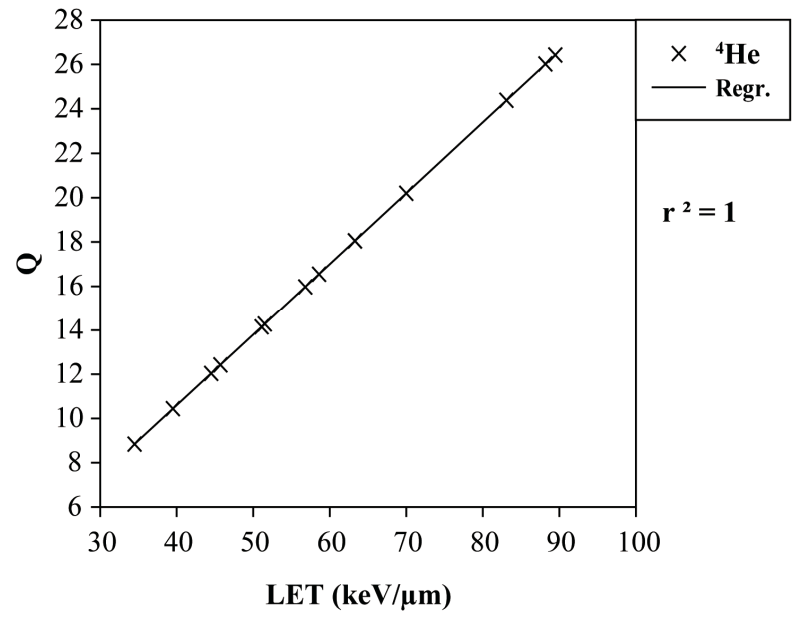

Figure 4. Relationship between quality factor and linear energy transfer for helium used to irradiate V79 cells invitro.

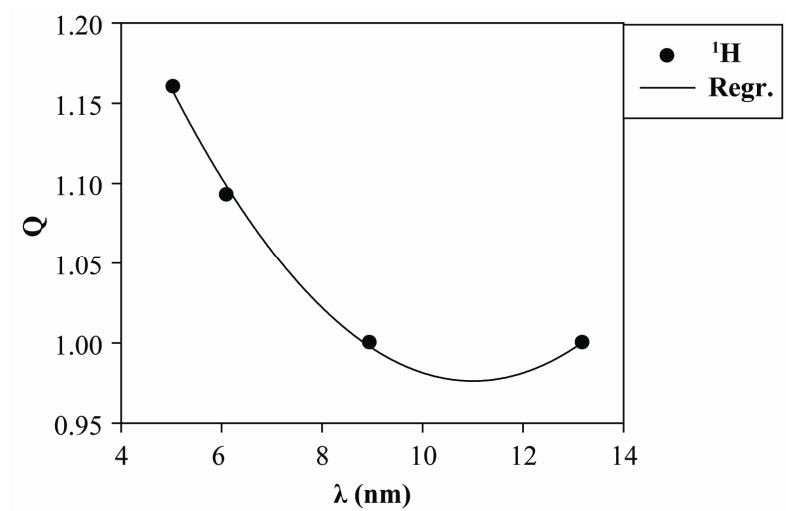

Figure 5. Relationship between quality factor and mean free path for protons used to irradiate V79 cells invitro. 
Figure 6 shows the relationship between quality factor and mean free path for helium particles, quality of effectiveness decreases with increasing of mean free path. The presented data is well fitted by the mathematical model which given as:

$$
Q(\lambda)=C_{14} \lambda+C_{15} \lambda^{2}+C_{16} \lambda^{3}+23.01
$$

where $C_{14}=-2.99, C_{15}=0.08$,

$$
C_{16}=8.99 \times 10^{-4} \text {, and } R^{2}=0.72 \text {. }
$$

Figure 7 gives relation between quality of effectiveness of protons and effective charge. The quality of effectiveness decreases dramatically as effectiveness charge increases until reach minimum value at $15 \times 10^{-4}$, after this value quality of inactivation by protons went up until the maximum at $4 \times 10^{-3}$ of effective charge. The mathematical model which describes these data is given as:

$$
\begin{aligned}
Q\left(Z_{\text {eff }}\right) & =C_{17} Z_{\text {eff }}+C_{18} Z_{\text {eff }}^{2}+C_{19} Z_{\text {eff }}^{3} \\
& +C_{20} Z_{\text {eff }}^{4}+C_{21} Z_{\text {eff }}^{5}+5.53
\end{aligned}
$$

where $C_{17}=-68.54 \times 10^{2}, C_{18}=25.47 \times 10^{5}$,

$$
C_{19}=96.59 \times 10^{-2}, C_{20}=-38.377 \times 10^{7},
$$$$
C_{21}=-1.13 \times 10^{13} \text {, and } R^{2}=0.97 \text {. }
$$

The Figure 8 represents the relation between quality factor of effectiveness for helium particles and effective charge. The quality decreases until reaches the minimum value, after that the quality increases constantly with increase effective charge reaching the maximum at $54.73 \times$ $10^{-4}$.

$$
\begin{aligned}
Q\left(Z_{\text {eff }}\right) & =C_{22} Z_{\text {eff }}+C_{23} Z_{\text {eff }}^{2}+C_{24} Z_{\text {eff }}^{3}+C_{25} Z_{\text {eff }}^{4} \\
& +C_{26} Z_{\text {eff }}^{5}+77.50 \times 10^{-2}
\end{aligned}
$$

where $C_{22}=15.78 \times 10^{-2}, C_{23}=12.77 \times 10^{-3}$,

$$
\begin{aligned}
& C_{24}=51.26 \times 10^{-2}, C_{25}=10.21 \times 10^{-2}, \\
& C_{26}=80.70, \text { with } R^{2}=0.89 .
\end{aligned}
$$

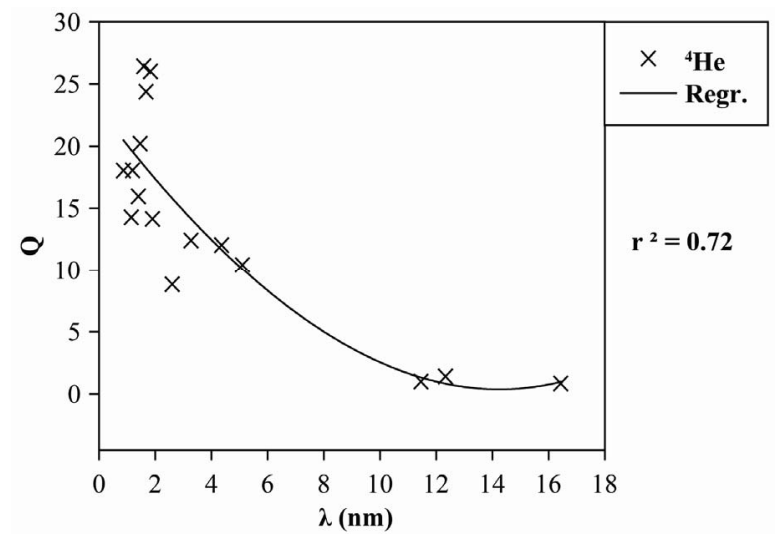

Figure 6. Relationship between quality factor and mean free path for helium particles used to irradiate V79 cells invitro.

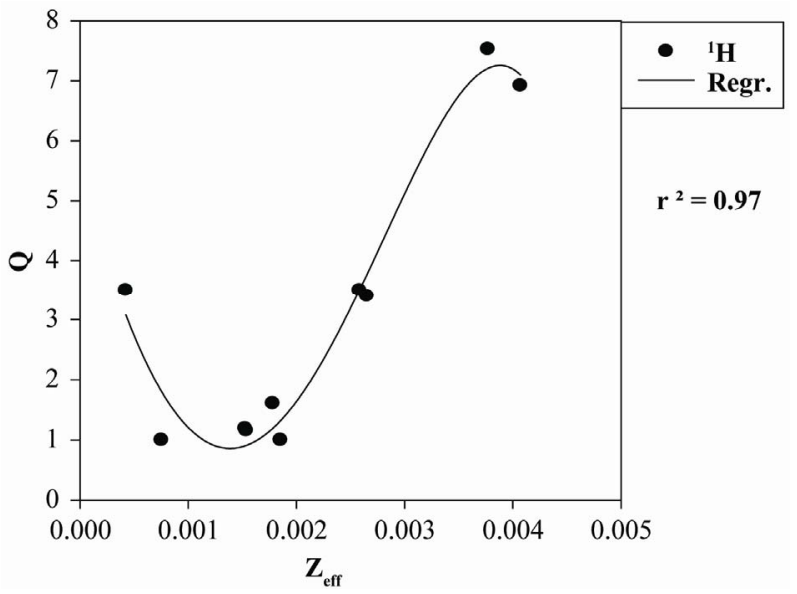

Figure 7. Relationship between quality factor and effective charge for protons used to irradiate V79 cells invitro.

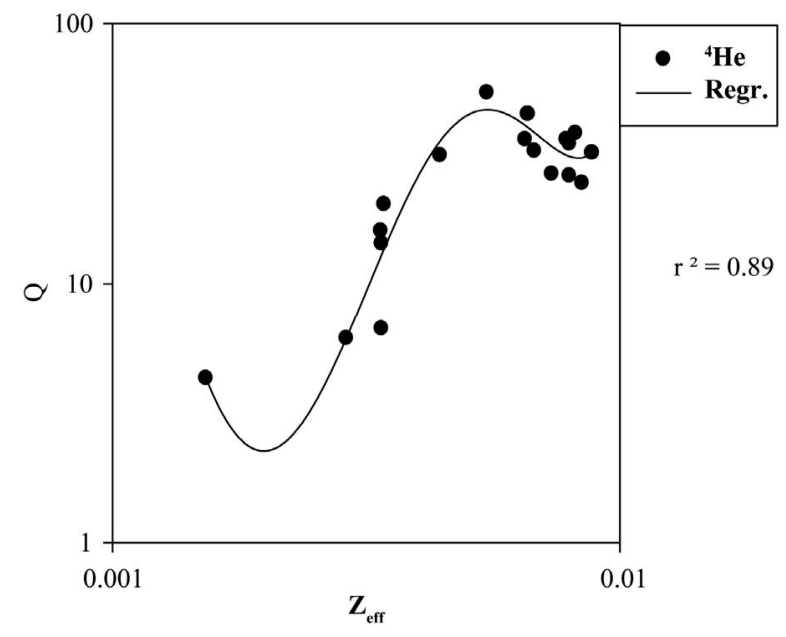

Figure 8. Relationship between quality factor and effective charge for helium used to irradiate V79 cells invitro.

\section{Conclusions}

Quality factor which represent the effectiveness of protons and helium particles has determined in terms of unrestricted linear energy transfer. Quantification of effectiveness for charged particles, i.e., protons and helium particles using mean free path and effective charge provide an alternative method to qualitative assess the effectiveness of ionizing radiations at lower doses.

The average distance between ionizations along the primary track of charged particles is more appropriate physical quantity than the absorbed or energy deposition parameters in quantifying the all types of biological effects of ionizing radiations in Micro-dimensional systems.

\section{Acknowledgments}

I truly acknowledge the school of applied physics for te- 
chnical assistant and guidance in carrying out this research.

I would like also extend my sincere gratitude to the Libyan High Education Committee for the financial support to make this research a successful one.

\section{References}

[1] W. K. Sinclair, "Experimental RBE Values of High Let Radiations at Low Doses and the Implications for Quality Factor Assignment,"Radiation Protection Dosimetry, Vol. 13, No. 1-4, 1985, pp. 319-326.

[2] D. E. Watt, C. H. Chen, L. A. Kadiri and A. Younis, "Towards a Unified System for Expression of Biological Damage by Ionizing Radiation," Health Effects of Low Dose Ionizing Radiation, British Nuclear Energy Society, Paper 6,1988, pp. 37-41.

[3] G. A. Nelson, "Fundamental Space Radiobiology," Gravitational and Space Biology Bulletin, Vol. 16, No. 2, 2003, pp. 29-36.

[4] J. Kiefer, "Biological Radiation Effects,” Springer-Verlag, Heidelberg, 1990.

[5] W. R. Hendee and F. M. Edwards, "Health Effects of Exposure to Low-Level Ionizing Radiation,” Medical Science Series, Institute of Physics Publishing Ltd., Bristol, 1996.

[6] E. J. Hall, "Radiology for Radiologist," 5th Edition, Lippincott Williams and Wilkins, Philadelphia, 2000, pp. 226-237.
[7] E. B. Saion, D. E. Watt, B. W. East and P. Colautti, “A Coaxial Double Cylindrical TEPC for the Microdosimetry of Selected Neutron Energy Bands in Mixed Fields of Fast Neutrons,” Radiation Protection Dosimetry, Vol. 31, No. 1-4, 1990, pp. 149-153.

[8] I. A. M. Al-Affan, "A New Concept in Microdosimetry to Evaluate the Quality Factor for Neutrons and Photons," Radiation Protection Dosimetry, Vol. 23, No. 1/4, 1988, pp. 83-86.

[9] D. E. Watt, "A Feasibility Study of Scintillator Microdosimetry for Measurement of the Biological Effectiveness of Ionizing Radiations," Radiation Protection Dosimetry, Vol. 61, No. 1-3, 1995, pp. 89-90.

[10] International Commission on Radiation Units and Measurements, "The Quality Factor in Radiation Protection," ICRU Publications, Bethesda, No. 40, 1986.

[11] International Commission on Radiation Units and Measurements, "Relative Biological Effectiveness, Radiation Weighting and Quality Factor," Annals of the ICRP Publication 92, Vol. 33, No. 4, 2003.

[12] R. J. Cannell and D. E. Watt, "Biophysical Mechanisms of Damage by Fastions to Mammalian Cells in Vitro," Physics in Medicine and Biology, Vol. 30, No. 3, 1985, pp. 255-258.doi:10.1088/0031-9155/30/3/006

[13] D. E. Watt, "Quantities for Dosimetry of Ionizing Radiations in Liquid Water," Taylor and Francis Ltd., London 1993. 